\section{Optical response of a single gold nanoparticle}

\section{N. Del Fatti, D. Christofilos* and F. Vallée}

FemtoNanoOptics Group, LASIM, Université Lyon1 and CNRS, 43 Bd. Du 11 Novembre, 69622

Villeurbanne, France

\begin{abstract}
Optical detection and spectroscopy of single gold nanoparticles using the spatial-modulation spectroscopy technique is described, focusing on the connection between the nanoparticle and environment parameters with the measured linear optical extinction spectrum. Characterisation of the size, shape and orientation on the substrate of a nanoparticle through quantitative determination of its optical extinction cross-section spectrum is illustrated in the case of gold nanospheres and nanorods. Extension of this technique to ultrafast nonlinear spectroscopy of a single gold nanoparticle is also discussed.
\end{abstract}

\section{Introduction}

The possibilities of designing and mastering the physical and chemical properties of nano-structured materials has led to considerable interest and activities in the academic and industrial domains. The impact of size reduction on these properties and the concomitant enhanced role of the interfaces are here key parameters. This is in particular the case for the linear and nonlinear optical properties of metal nanoobjects, for which size reduction leads to the appearance of a pronounced resonance in their optical response, the well known surface plasmon resonance, often also referred as the Localised Surface Plasmon Resonance (LSPR) in nanoparticles [1-4]. To a large extent its spectral properties, wavelength and width, can be adjusted by tailoring the particle shape (sphere, rod, disk, triangle, cube, cage, ...), size, composition (bi-metallic or hybrid particles), structure (core-shell) and environment (solid or liquid matrix, porosity or anisotropy of the surrounding material, bound molecules, presence of other nanoobjects, ...) [3-12]. The latter dependence makes metal nanoparticles large potential tools as optical nanosensors of their local environment, over a range of about their size, i.e., much smaller than the optical wavelength [3,10-12]. Therefore, proper design of a metal nanoparticle size and shape and detailed modelling of their effects on its properties are important problems in nanosciences and nanotechnology, with direct impact in the development of plasmonic nanomaterials or on their use as chemical and biological sensors. In this context, gold is one of the most studied materials due to many advantages such as chemical stability, catalytic activity of small size particle, and biocompatibility.

Most investigations were performed on large ensemble of particles dispersed in a liquid or solid matrix or deposited on a substrate, yielding a good understanding of their linear and nonlinear optical responses [2,3,8,9,13]. However, detailed and quantitative interpretation of the obtained data and their precise comparison with theoretical models are hampered by the unavoidable nanoparticle size, shape and environment fluctuations of the synthesised nanoobjects. Precise determination of their properties and correlation with the theoretical models as well as technological developments, such as nanometric resolution sensors, require investigation of a single object.

Detection of a single luminescent nanoobject is now a routine experiment applied to study their individual properties or use them as labellers [14-16]. Observation of single veryweakly luminescent objects such as metallic nanoparticles is more difficult. The high spatial resolution of the near field optical technique (SNOM) has first been exploited to detect single gold nanoparticles $[17,18]$. The complexity of these studies and of their interpretation due to tip-particle interaction [19], has fostered the development of far-field techniques. It requires either detection of its direct effect on the incident light, i.e., weak absorption or scattering, or observation of another parameter indirectly impacted by light absorption. 
The first investigations of a single metal nanoparticle were performed by detecting their light scattering [20-25]. As scattering efficiency decreases with the square of the particle volume $V[3,4]$, these studies are limited to relatively large particle sizes, diameters larger than $20 \mathrm{~nm}$ for gold nanospheres (and down to about $5 \mathrm{~nm}$ using heterodyne detection [24]). As absorption scales as $V$, it strongly dominates over scattering for small particles and is then the indicated parameter to monitor (the absorption cross-section of a $20 \mathrm{~nm}$ gold nanosphere at its LSPR wavelength is about 100 times larger than the scattering one). Absorption based detection of a single gold particle has been performed indirectly, via monitoring the induced local heating of the particle and its environment [26]. Direct detection of the farfield optical extinction of a single metal (gold) nanoparticle has been recently reported using a spatial-modulation spectroscopy (SMS) technique [27-29]. This is based on spatial modulation of the position of the studied particle and detection of the concomitant modulation of the transmitted light energy. Though extinction is measured, for a small particle, the scattering contribution can be neglected and the measured signal directly connected to its absorption. Compared to other approaches, it has the large advantages of permitting a quantitative determination of the single nanoparticle cross-section, of requiring only a moderate brightness source, and of being easily combinable with nonlinear optical measurements [30].

In this paper, we discuss results obtained with the SMS technique in single gold nanoparticles with different shapes: quasi-spheres and rods [27,29,31]. After introducing the SMS technique, we discuss optical characterisation of a single gold nanoparticle (determination of its size, geometry and surface orientation) and of its local environment by comparison of its measured and calculated spectra. Finally, its application to nonlinear optical study of a characterised single gold nanoparticle is introduced, based on the combination of SMS with ultrafast nonlinear spectroscopy.

\section{Spatial Modulation Spectroscopy (SMS)}

For a light beam of power $P \mathrm{i}$ incident on a small particle, i.e., of size much smaller than the beam diameter, localised at point $(x, y)$ in the plane perpendicular to the beam propagation, the beam extinction is given by

$$
\Delta P=P \mathrm{i}-P \mathrm{t}=\sigma_{\mathrm{ext}} I(x, y)
$$

where $I(x, y)$ is the intensity spatial profile in the particle plane and $P t$ the transmitted power. The extinction cross-section $\sigma_{\text {ext }}=\sigma_{\mathrm{abs}}+\sigma_{\mathrm{sc}}$ of the nanoparticle can be identified with its absorption cross-section $\sigma_{\text {abs }}$ for small sizes (less than about $30 \mathrm{~nm}$ for a gold sphere). For a single $20 \mathrm{~nm}$ gold nanosphere in water the peak value of the latter in the visible range, i.e., at the LSPR wavelength, is of the order of $\sigma_{\mathrm{abs}} \approx 300 \mathrm{~nm}^{2}$. About $10^{-3}$ of the incident light power will be absorbed for a light beam focused to a $500 \mathrm{~nm}$ diameter focal spot. This is beyond the sensitivity limit of conventional spectroscopic techniques, but can be easily detected using lock-in detection methods, provided that this absorbed fraction is modulated. This is the main concept of the Spatial Modulation Spectroscopy technique we recently developed: the position of the nanoparticle is modulated in the focal plane of the

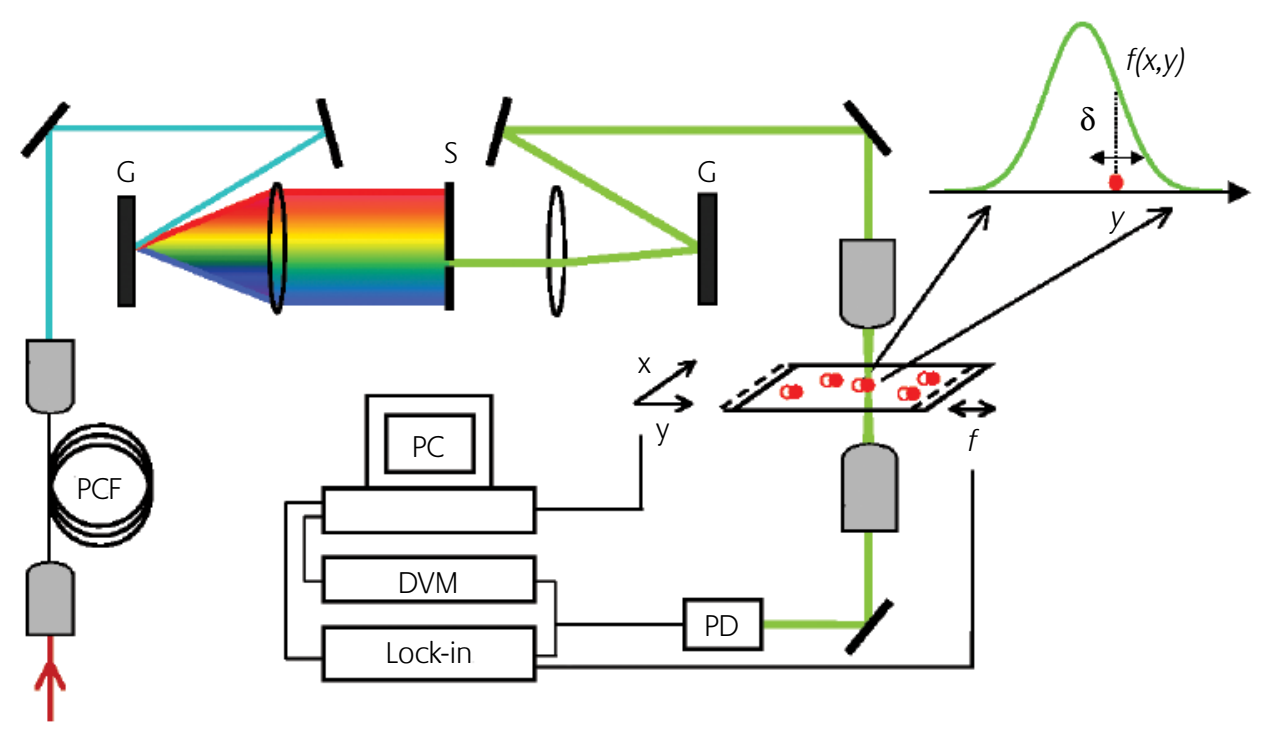

\section{Figure 1}

Schematic of the single-particle spatial modulation spectroscopy (SMS) set-up, showing the transmission microscope formed by the focusing microscope objective, a piezo-electric element modulating the sample position at frequency $f$, the $x, y$ scanner, the collecting objective, the Si photodiode (PD), the digital voltmeter (DVM), and the lock-in amplifier demodulating the signal. A tuneable light source is created from a supercontinuum generated in a photonic crystal fibre (PCF) frequency filtered using a grating pair (G) and a slit (S) 


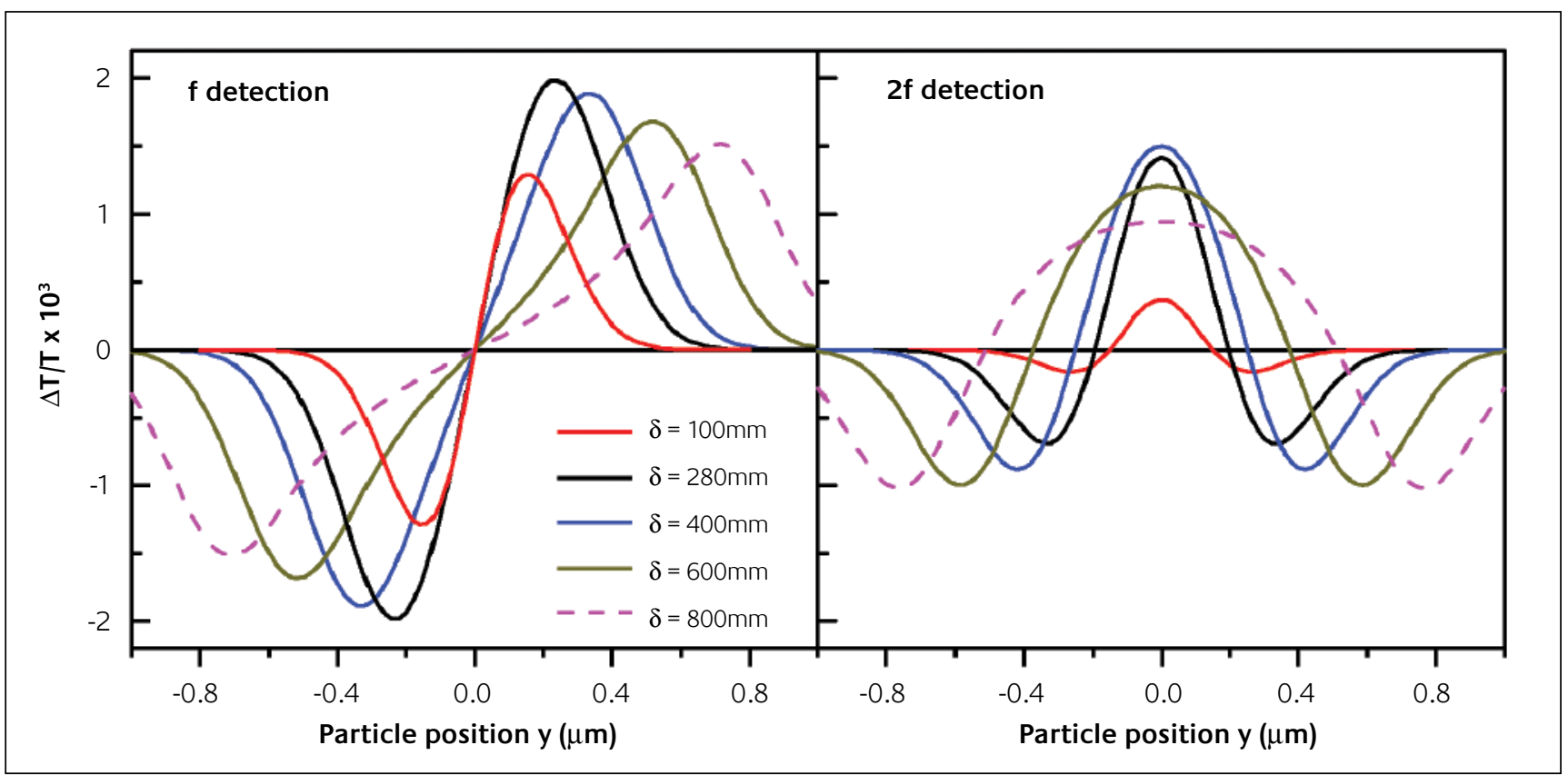

\section{Figure 2}

Calculated relative transmission change $\Delta T / T$ modulated at $f$ or $2 f$ for a Gaussian laser beam focused at $(x=0, y=0)$ with $d=0.34 \mu m$, as a function of the position of the nanoparticle along the $y$ direction $(x=0, y)$. The extinction cross-section of the nanoparticle has been assumed to be $\sigma_{\text {ext }}=290$ nm ${ }^{2}$ (corresponding to that of a $D=20 \mathrm{~nm}$ gold nanosphere in water for a wavelength $\lambda$ slightly off resonance with its $L S P R$ ). The different curves are obtained for a modulation in the y direction with amplitude $\delta=100 \mathrm{~nm}, 280 \mathrm{~nm}, 400 \mathrm{~nm}, 600 \mathrm{~nm}$ and $800 \mathrm{~nm}$

light beam, which results in a modulation of $P_{\mathrm{t}}$, i.e., of the measured sample transmission (Fig. 1).

If the modulation amplitude $\delta$ is much smaller than the beam size, the absorbed power $\Delta P$ can be expanded in $\delta$ yielding, up to the second order [27]:

$\Delta P \approx \sigma_{e x t}\left[I(x, y)+\delta \frac{\partial I(x, y)}{\partial y} \sin (2 \pi f t)+\frac{\delta^{2}}{2} \frac{\partial^{2} I(x, y)}{\partial y^{2}} \sin ^{2}(2 \pi f t)\right]$

assuming that modulation takes place at frequency $f$ along $y$. When recovering the modulated transmission at the fundamental, $f$, or second harmonic, $2 f$, frequency a signal proportional to the first or second derivatives of $l(x, y)$ is obtained, respectively. In both cases, quantitative analysis of the modulated part of the transmitted light yields the absolute value of the single-particle extinction cross-section $\sigma_{\text {ext }}$ provided the modulation amplitude and the beam profile are known [27].

In experiments large modulation amplitudes (comparable to the beam size) are used and the transmitted power has to be calculated numerically. The results of such calculation performed for a Gaussian beam profile I $(x, y)$ with a full-widthat-half-maximum $d$ are shown in Fig. 2. As expected, the spatial profiles of $\Delta T / T=-\Delta P / P_{i}$ demodulated at $f$ or $2 f$ show derivative-like profiles, similar to the very small amplitude regime (2). However, significant distortions are observed as $\delta$ becomes comparable to the beam diameter. Concomitantly, the $\Delta T / T$ amplitude decreases (Fig. 2). A maximum of the transmission change is obtained for optimal modulation amplitudes of $\delta_{\text {opt }}^{f} \approx 0.8 d$ and $\delta^{2 f}{ }_{\text {opt }} \approx d$ for $f$ and $2 f$ detection, respectively. The spatial resolution of the set-up is set by both the focal spot size $d$ and by the modulation amplitude. It can be quantified by the distance between the extrema of $|\Delta T|$ $\mathrm{T} \mid$ or its two side peaks for $f$ and $2 f$ detection, respectively (Fig. 2). It remains almost constant (and set by $d$ ) for small $\delta$, and increases as $\delta$ reaches the beam size. As for the optimal amplitudes $\delta_{\text {opt }}^{f}$ or $\delta^{2 f}{ }_{\text {opt }}$, spatial resolution is only slightly degraded, these values are systematically used in SMS measurements.

The experimental set-up for SMS measurements is schematically shown in Fig. 1. The incident light is focused onto the sample surface using a 100x microscope objective with Numerical Aperture 0.8. The sample is periodically translated in the focal plane at a frequency of about $1.5 \mathrm{kHz}$ by means of a piezoelectric element. As in all far-field studies, the limited spatial resolution (roughly half the optical wavelength) is overcome by using diluted systems. Their surface density is typically less than one particle per $\mu \mathrm{m}^{2}$, so that only one particle is in the optically probed zone. They were prepared by spincoating very diluted nanoparticle colloidal solutions onto a glass substrate. The transmitted light is collected using an identical objective and detected by a silicon photodiode. The modulated part of the transmission $\Delta T$ is demodulated by a lock-in amplifier and the total sample transmission $T$ simultaneously measured using a digital voltmeter. The sample is mounted onto a $x$-y piezoelectric translation stage to record two-dimensional images of the sample surface with a $\Delta T / T$ sensitivity of the order of $10^{-5}$. Absorption being directly detected, $\Delta T / T$ is independent of $P_{i}$. Therefore, very low powers can be used (down to less than 1 $\mu \mathrm{W}$ ) which avoids sample damaging and permits the use of low power sources [27,29,32,33]. 
(a)

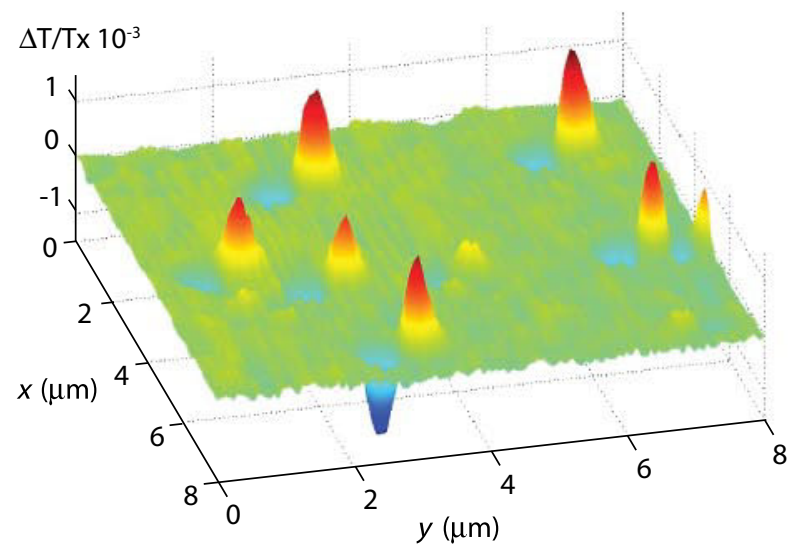

(b)

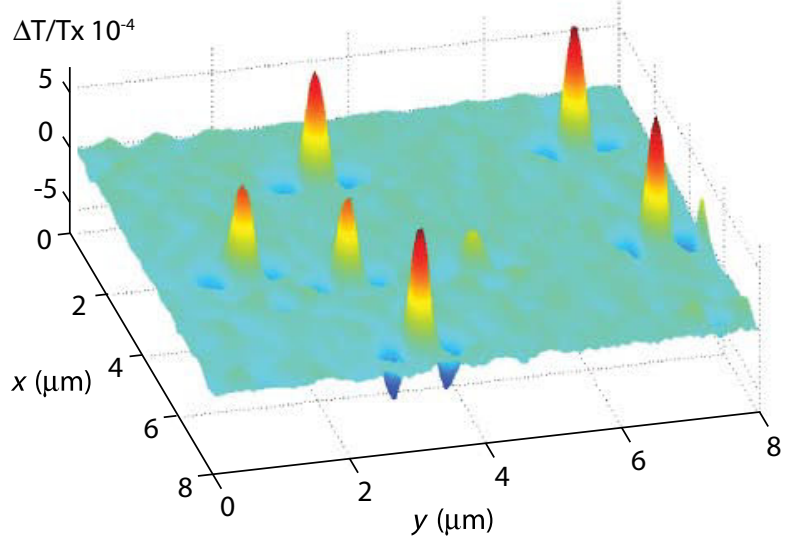

\section{Figure 3}

Spatial $x, y$ dependence of the transmission change $\Delta T / T$ detected at $f(a)$ and $2 f(b)$. Each structure corresponds to a single gold nanoparticle, lying in between the two-extrema and at the peak maximum for $f$ and $2 f$ detection, respectively (the mean particle diameter is $<D>\approx 20$ mm). The laser, with wavelength $\lambda=532 \mathrm{~nm}$ and power $P_{i}=10 \mu \mathrm{W}$, is focused on a $d=0.34 \mu \mathrm{m}$ focal spot. For all images, the sample position is scanned over the same $8 \times 8 \mu \mathrm{m}^{2}$ zone and modulated along the $y$ axis at $f=1.5 \mathrm{kHz}$ with $\delta \approx 0.3 \mu \mathrm{m}$

\section{Single gold nanoparticle extinction cross-section}

Detection of gold nanospheres with mean diameter $20 \mathrm{~nm}$ is illustrated in Fig. 3 for $f$ and $2 f$ detection (a laser source at $532 \mathrm{~nm}$ has been used as light source). Each feature in the modulation direction $y$, i.e., negative and positive extrema for $f$ detection and main peak with two negative satellites for $2 f$, is associated to a single gold nanoparticle. The $\Delta T / T$ signal shape along the transverse direction, $x$, reflects the intensity spatial profile of the light beam at the focal spot (Fig. 4). It thus provides a direct reading of the spot size that can be used as input for fitting the experimental data (provided that astigmatism is negligible). The modulation amplitude influences the signal shape along the $y$ direction, but only weakly its amplitude provided that $\delta$ values close to $\delta^{\mathrm{f}, 2 f}$ opt are used (Fig. 2).

The amplitude of the measured $x-y$ profiles is thus determined by the single particle extinction cross-section permitting its quantitative determination. It has been measured for a gold nanosphere with diameter down to $5 \mathrm{~nm}$ at $\lambda=532 \mathrm{~nm}$, close to their surface plasmon resonance wavelength, about $520 \mathrm{~nm}$ [27]. Actually such a full $x, y$ signal analysis procedure is not required if the focal spot size is first precisely calibrated. As stressed above, this can be done using a single nanoparticle as a probe, the maximum $\Delta T / T$ amplitude at $f$ or $2 f$ then directly translating into the $\sigma_{\text {ext }}$ value $[28,29]$.

The absorption cross-section of a nanoparticle being proportional to its volume $[3,4]$, the observed $\Delta T / T$ amplitude for a nanoparticle from a given sample with mean diameter $<$ D $>$ fluctuates from particle to particle due to their size dispersion. In each sample, the statistics of the maximum amplitudes shows a monomodal distribution with a dispersion in quantitative agreement with the TEM measured size distribution of the initial colloidal solution (Fig. 5). This agreement, together with the absence of any weak signal out of the distribution, confirms that single nanoparticles are observed. Experiments performed in different samples with mean diameter ranging from 5 to $20 \mathrm{~nm}$ show that the mean $\Delta \mathrm{T} / \mathrm{T}$ amplitude and thus the mean $\sigma_{\text {ext }}$ is almost proportional the particle volume in agreement with

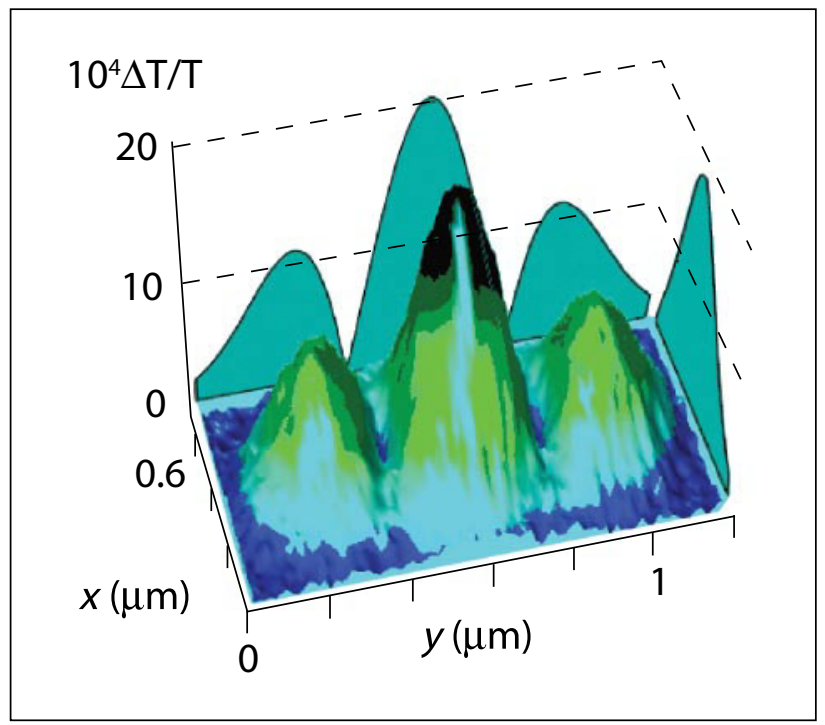

\section{Figure 4}

Two-dimensional image of a single gold nanoparticle taken at $2 f$ using $\lambda=520 \mathrm{~nm}$ and $\delta=0.28 \mu \mathrm{m}$ (the absolute value of the measured $\Delta T / T$ is shown). The projections onto the axis planes show the gaussian beam profile $(x)$ and the modulation signal shape $(y)$. Their fits yield the beam size $d=0.35 \mu \mathrm{m}$ and the particle extinction cross-section $\sigma_{\text {ext }}=450 \pm 20 \mathrm{~nm}^{2}$ 


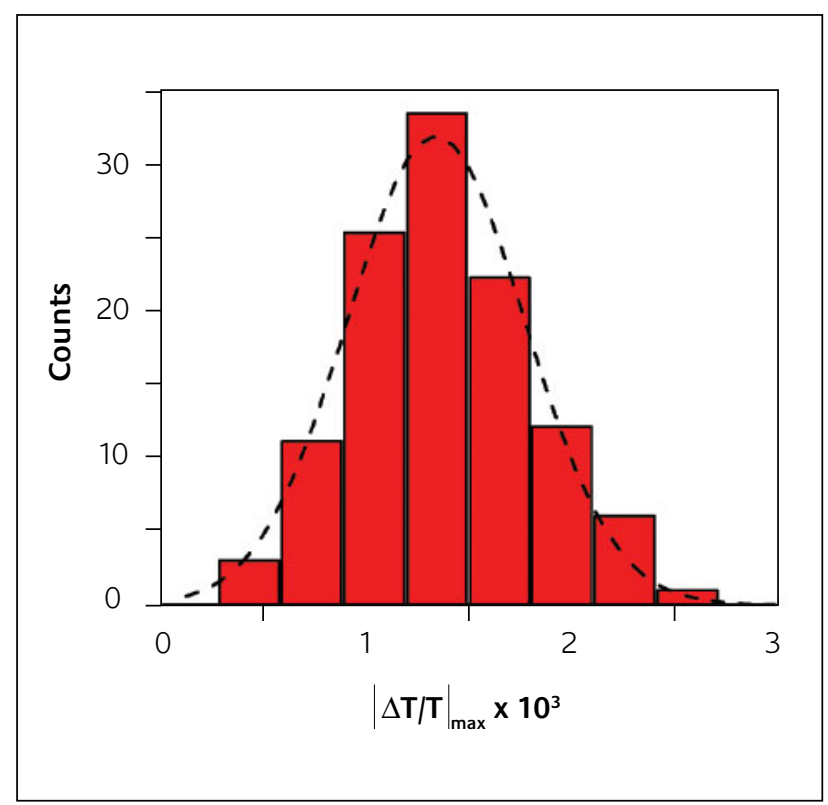

Figure 5

Histogram of the maximum signal amplitude distribution measured at $\lambda=532 \mathrm{~nm}$ with $f$ detection for the gold nanoparticle sample with average size $\langle D>=20 \mathrm{~nm}$. The dotted line is a Gaussian fit to the data. The standard deviation is about $30 \%$ of the average signal in agreement with the TEM measured particle volume distribution

nanoparticle extinction dominated by absorption for the studied size range. Furthermore, the measured $\sigma_{\text {ext }}$ values are in good quantitative agreement with the computed ones for gold nanoparticles deposited on glass using the average medium approach, i.e., using a mean air-glass dielectric constant to characterise the dielectric environment of the nanoparticle [27].

\section{$4 \quad$ Single gold nanoparticle absorption spectroscopy}

Combining the above spatial modulation microscopy system with a tuneable light source, extinction spectrum of a single-nanoparticle can be measured. As only a mediumbrightness source (typically a few $\mu \mathrm{W}$ in the selected bandwidth of $3 \mathrm{~nm}$ ) is required for SMS measurement, the broadly tuneable visible light can be provided by supercontinuum generation in a photonic crystal fibre [27,28] or even a white-lamp source if only a medium sensitivity is necessary (i.e., for large nanoparticles, typically $D \geq 20 \mathrm{~nm}$ ) [32,33]. The results presented below were obtained using the former system, injecting the fibre with the $20 \mathrm{fs}-780 \mathrm{~nm}$ pulse train of a home-made Ti:sapphire femtosecond oscillator operating at $80 \mathrm{MHz}$ repetition rate. The fibre generated supercontinuum [34], is dispersed by a grating pair separated by a -1 magnification telescope (Fig. 1). It is frequency filtered by a slit placed close to the focal plane and adjusted to select a $3 \mathrm{~nm}$ bandwidth [35]. This provides an unpolarised light source easily tuneable from the near infrared to about $450 \mathrm{~nm}$.

\subsection{Optical absorption of a quasi-spherical gold nanoparticle}

The extinction spectrum of a single gold nanoparticle around its LSPR is illustrated in Fig. 6. For a given polarisation it exhibits the characteristic shape expected for quasi-spherical small gold particles, i.e., an enhanced absorption due to surface plasmon resonance (around $530 \mathrm{~nm}$ ) overlapping the rise of the interband absorption that dominates on the blue side of the spectrum $[3,9,28]$. In systematic experiments, this characteristic spectral profile is used to crudely select the studied nanoobject. For instance, in the case of gold nanospheres, this is done by checking the relative change of the $\sigma_{\text {ext }}(\lambda)$ amplitude for a wavelength either close to the LSPR or away from it and the interband absorption, in the red part of the spectrum (Fig. 6). This two-wavelength test permits to quickly select objects for full spectroscopic investigation, and to reject the small fraction of spurious absorbers as dust particles or aggregates.

The measured spectra exhibit large dependencies on the linear polarisation direction of the incident light, with correlated extrema of the amplitude and LSPR wavelength for perpendicular polarisation directions (Fig. 6). This dependence on the polarisation direction is further illustrated by the polar plot of $\sigma_{\text {ext }}$ at $550 \mathrm{~nm}$ clearly showing maximum and minimum $\sigma_{\text {ext }}$ amplitudes along the $60^{\circ}$ and $150^{\circ}$ directions, respectively (inset of Fig. 6). Such polarisation

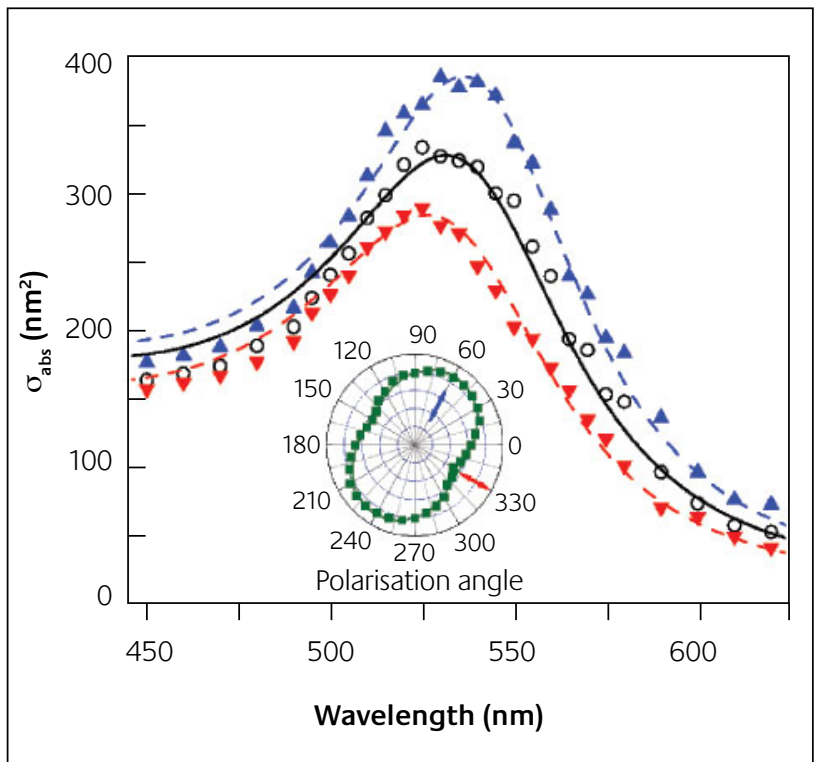

\section{Figure 6}

Extinction spectrum of a single gold nanoparticle measured for unpolarised (black dots) and linearly polarised light along the main particle axes in the surface plane (red and blue triangles for light polarised along the $150^{\circ}$ and $60^{\circ}$ polarisation direction, respectively). Lines denote fits using the spherical (full line) and elliptical (dashed lines) particle approximations, respectively for unpolarised and linearly polarised light. The optically deduced particle diameter is $D=18.5 \mathrm{~nm}$ and its aspect ratio $\eta=0.92$. The inset shows the polarisation dependence of the absorption cross-section $\lambda=550 \mathrm{~nm}$, with red and blue arrows denoting the particle main axes in the surface plane. The line is a fit using a superposition of two perpendicularly oriented dipolar absorption spectra 
dependent LSPR behaviour is the optical signature of deviation of the particle from a spherical shape $[3,4,9,28]$. It has been used to extract quantitative information on the particle deviation from sphericity, assuming it takes a slightly elliptical shape.

The absorption cross-section of a small ellipsoid (much smaller than the optical wavelength) of dielectric constant $\varepsilon=\varepsilon_{1}+i \varepsilon_{2}$ embedded in an homogeneous medium with dielectric constant $\varepsilon_{\mathrm{m}}$, can be analytically calculated using the quasistatic approximation $[3,4,28]$. For light linearly polarised along the i main axis of the ellipsoid of major axis half-length $a, b$ and $c$ (with $a \geq b \geq c$ ), it reads:

$$
\sigma_{a b s}(\lambda)=\frac{2 \pi V \varepsilon_{m}^{3 / 2}}{\lambda L_{i}^{2}} \frac{\varepsilon_{2}(\lambda)}{\left|\varepsilon(\lambda)+\varepsilon_{m}\left(1-L_{i}\right) / L_{i}\right|^{2}}
$$

where $V$ is the particle volume. The geometrical factors $L_{i}$ depend on the particle geometry [4]. They reduce to $1 / 3$ for a sphere leading to the well-known expression of the absorption of a small nanosphere keeping only the dipolar term in the Mie theory $[3,4]$. The metal dielectric function in the nanoparticle $\varepsilon$ can be approximated by its bulk gold dielectric function modified by introducing a surface scattering term [3]. As the latter also impacts the shape of the measured spectrum, broadening the LSPR, it can also be extracted from fitting the experimental data [36]. Actually, for sufficiently large nanoparticles (size larger than typically 10 - $20 \mathrm{~nm}$ ), it plays a weak role and won't be discussed here where we will focus on the main parameter determining the spectrum and amplitude, i.e., the particle shape and volume and the dielectric constant of its environment.

The shape of the nanoparticle spectrum is essentially determined by the local field enhancement effect (second term of the right hand side part of (3)) [3,13]. It is thus set by the environment of the particle, through $\varepsilon_{\mathrm{m}}$, and its geometry through $L_{\text {i. }}$ In particular, as a good approximation, the surface plasmon resonance wavelength $\lambda_{\text {SPR }}^{i}$ for light polarised along the $i$ direction corresponds to minimising the denominator in (3). For a weak dispersion of the imaginary part of the metal dielectric constant $\varepsilon_{2}$, it corresponds to the condition $[3,4,28]$ :

$$
\varepsilon_{1}\left(\lambda_{S P R}^{i}\right)+\varepsilon_{m}\left(1-L_{i}\right) / L_{i}=0
$$

yielding three LSPR polarised along the ellipsoid main axes.

As in transmission electron microscopy (TEM) measurements, only the particle anisotropy in the $x, y$ plane is detected and one has to make an assumption about its size in the $z$ direction. The simplest one consists in assuming that it is the same as in the $x$ or $y$ direction, i.e., that the particle is a prolate $(a>b=c)$ or oblate $(a=b>c)$ spheroid. Only two LSPR polarised along the short and long spheroid axes are

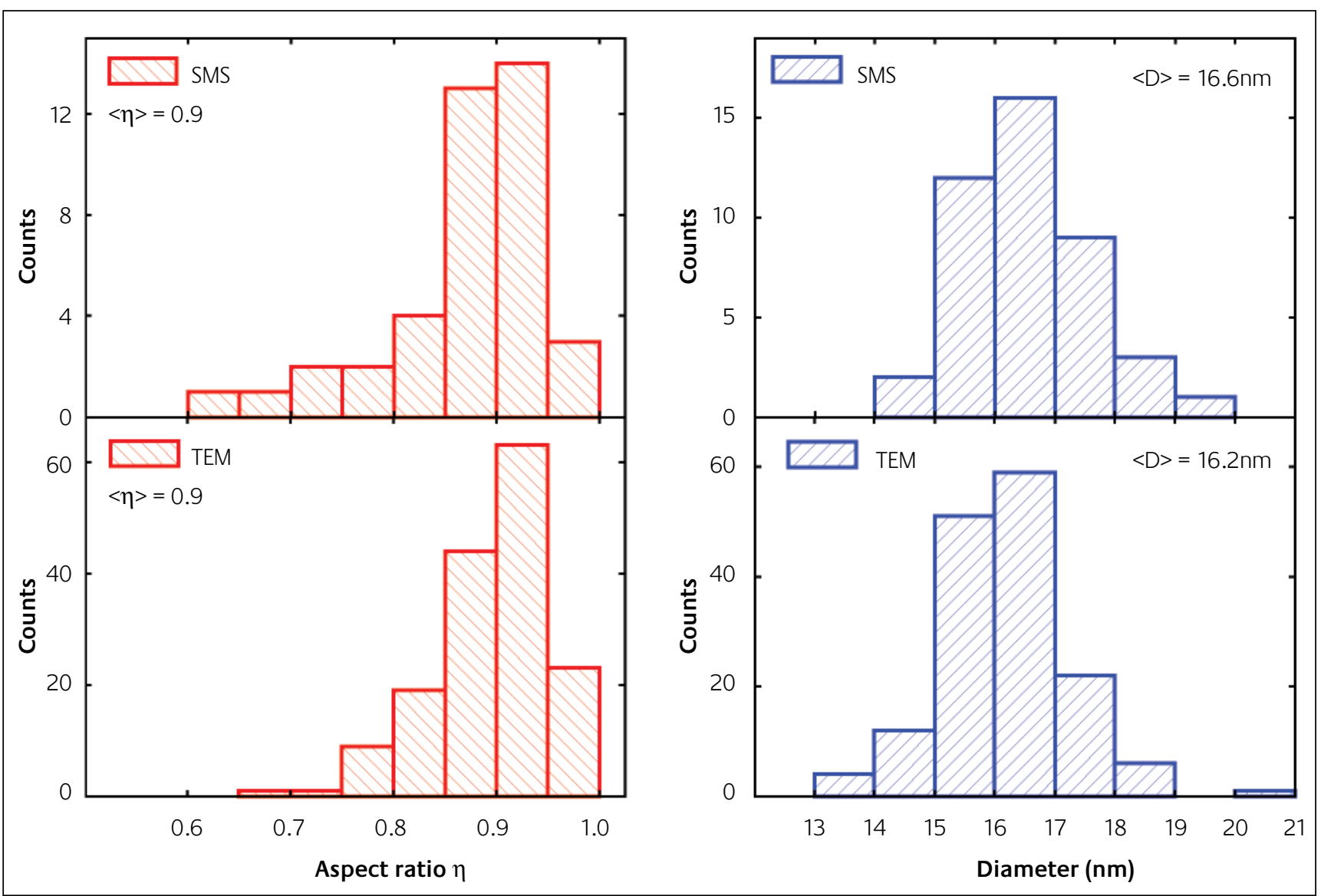

Figure 7

Statistics of aspect ratio and diameter deduced from SMS optical measurements and from electron microscopy (TEM) for quasi-spherical gold nanoparticles. The mean value of the aspect ratio and diameter are $\langle\eta\rangle=0.9$ and $D=16.6 \mathrm{~nm}$ for $S M S$ and $\langle\eta>=0.9$ and $D=16.2 \mathrm{~nm}$ for TEM measurements 
thus obtained with $L_{i}$ only depending on the spheroid aspect ratio $\eta=c / a$.

In this model, the main polarisation directions determined in SMS investigation of a single nanoparticle are associated to its main axes in the substrate plane [29]. The aspect ratio $\eta$ directly translates into the maximum LSPR wavelength displacement, $\Delta \lambda_{\text {SPR }}^{\text {l-s }}$, with light polarisation (i.e., for polarisation along the short (s) or long (I) particle axis). This permits its precise determination from the experimental shift for a given shape assumption (all the other parameters in (3) and (4) being light polarisation independent). Actually, for a weakly elliptical nanoparticle (i.e., $\eta \approx 1), \Delta \lambda_{\text {ISR }}^{\text {ls }}$ can be estimated by expanding the geometrical factors $L_{i}$ in power of $(1-\eta)$ around it sphere value $\left(L_{i}^{s p h}=1 / 3\right)$. Developing the resonance condition (Eq. 4) around the LSPR wavelength of a sphere, $\lambda_{\text {Sph }}^{\text {spR }}$, one obtains:

$$
\Delta \lambda_{S P R}^{l-s}=-\frac{54}{15}(1-\eta) \varepsilon_{m} /\left(\frac{\partial \varepsilon_{1}}{\partial \lambda}\right)_{\lambda s p h}
$$

It is independent of the assumed shape permitting unambiguous $\eta$ extraction. This is confirmed by the fact that the distribution and mean value of the aspect ratio deduced from optical polarisation studies are in excellent agreement with that obtained from TEM measurements on particles from the same batch solution (Fig. 7).

The LSPR wavelength value is mostly determined by the dielectric constant of the surrounding material. It can thus be determined from the measured spectra but slightly depends on the assumed oblate or prolate shape. For instance, for a small ellipticity, switching from the former to the latter assumption leads to reduction of the estimated $\varepsilon_{\mathrm{m}}$ value by about $3(1-\eta) \varepsilon_{\mathrm{m}} / 5$ (yielding a relative change of about $6 \%$ for the mean value of $\varepsilon_{\mathrm{m}}$ for $\left.\langle\eta\rangle=0.9\right)$. Since the particle shape cannot be unambiguously determined, this translates into an uncertainty in the extracted $\varepsilon_{\mathrm{m}}$. Note that the LSPR being sensitive to dielectric environment of the nanoparticle over a distance of the order of its size, this $\varepsilon_{\mathrm{m}}$ value corresponds to a local value, in the close vicinity of the particle [36].

Such measurements open-up the possibility of using small gold nanoparticle as quantitative nanoprobe of the dielectric properties of a material. This has been illustrated in the case of gold nanospheres performing measurements on the as spin-coated sample or after adding a drop of polymer [36]. In the former case, the particles are surrounded by air and the glass substrate and a mean environment refractive index $<n_{m}>$ of 1.34 is deduced. In the latter case where they are embedded into a polymer layer, $<n_{\mathrm{m}}>$ increases to 1.44 , a value consistent with the polymer refractive index [36]. In both cases, large particle to particle fluctuations with standard deviation of 0.07 and 0.09 were observed. The obtained result gives direct experimental evidence for fluctuations of the local environment (including the remaining surfactant molecules bound at the particle surface) on a nanometric scale within the same composite medium. It demonstrates the possibility of using nanoabsorbers as probes of their nanoenvironment, with a potential sensitivity to both the real and imaginary parts of the local refractive index on a nanoscale.

The nanoparticle volume $V$ is a key parameter in the absorption cross-section amplitude (3). As quantitative measurements are performed with the SMS technique, it can also be determined through reproducing the $\sigma_{\mathrm{abs}}$ overall amplitude (conversely to other single particle optical experiments) [29]. As for the environment dielectric function, the deduced value depends on the assumed particle shape. Characterising the particle size by its equivalent diameter $D_{\text {eq, }}$, defined as that of a sphere of same volume, values differing by about $2 \%$ were obtained for prolate or oblate shapes. This is within our experimental error permitting reliable extraction of $D_{\text {eq }}$. SMS polarisation dependent spectra thus permit a full optical characterisation of a single metal nanoparticle: its volume, and in plane anisotropy and orientation are determined.

For quasi-spherical nanoparticles $(<\eta>\approx 0.9)$, the particle size and environment dielectric function can be more easily extracted performing SMS measurements with unpolarised light. As information on particle anisotropy is lost, the measured spectra can be fitted assuming a nanosphere, i.e., using (3) with $L_{i}=1 / 3$, as illustrated in Fig. 6. The extracted parameters, i.e., $\eta_{\mathrm{m}}$ and particle diameter $D$, then lies between those deduced from full analysis of the polarisation dependent data using the prolate or oblate shape assumption. As long as only these parameters are required, such measurements are thus sufficient. The statistics of the gold nanoparticle diameter extracted using this approach is compared to the TEM results in Fig. 7. As for the particle aspect ratio, excellent agreement between the SMS and TEM data is obtained (in particular, the mean diameter differs by less than 3\% for the particles investigated in Fig. 7) [29].

\subsection{Optical extinction of a single gold nanorod}

The above SMS technique can be extended to other nanoparticle shapes as illustrated in Fig. 8 for gold nanorods. As in an ellipsoid [28], shape anisotropy lifts the LSPR degeneracy leading to strongly polarisation dependent LSPR [37-39]. Its longitudinal LSPR, polarised along its long axis, is strongly red shifted as compared to the sphere case [37-39]. In contrast its transverse LSPR, polarised in the plane perpendicular to its long axis, only slightly blue shifts (Fig. 8). The ensemble absorption spectrum of a nanorod aqueous solution thus exhibits two main resonances, with, because of orientational averaging, no light polarisation effect [37-39]. This is illustrated in Fig. 8 for an ensemble of gold nanorods with length $L$ in the 40 to $60 \mathrm{~nm}$ range and a width $D$ between 15 and $20 \mathrm{~nm}$. The corresponding aspect ratios $\eta=D / L$ range from 0.25 to 0.5 , much smaller than for the quasi-spherical case (section 4.1). It is thus associated to much larger LSPR shift, with an ensemble averaged longitudinal LSPR wavelength around $680 \mathrm{~nm}$, and a second transverse resonance at 515 $\mathrm{nm}$ with a much weaker amplitude (due to overlap with the gold interband transition). 


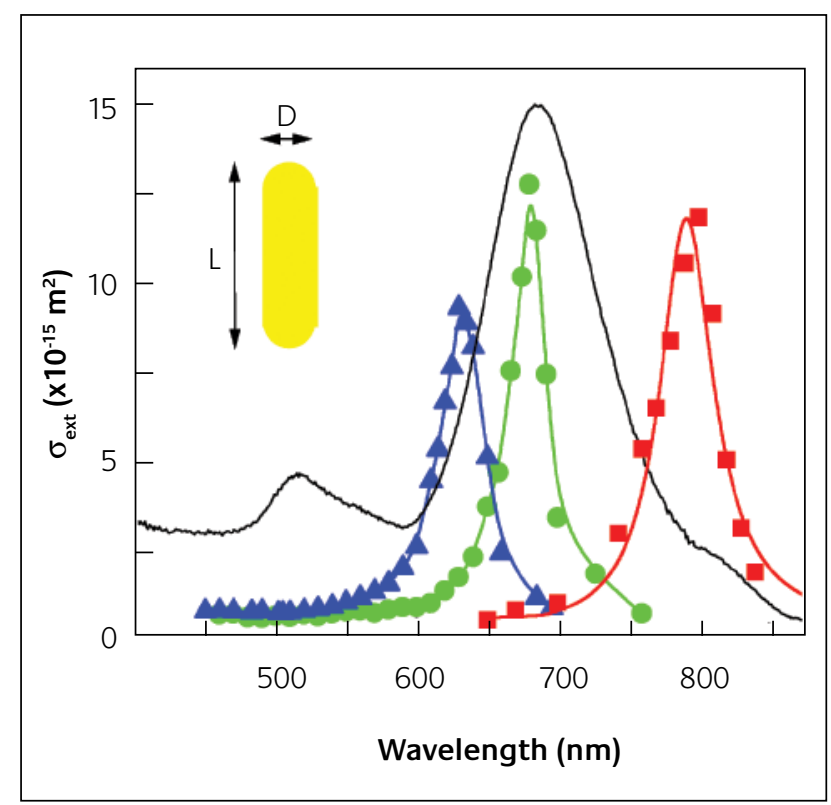

Figure 8

Absolute extinction cross-sections spectra of three single gold nanorods of different aspect ratio $\eta=D / L$ embedded in $\mathrm{PVOH}$ ( $\eta$ is estimated to be about $0.5,0.35$ and 0.25 for the triangles, dots and squares, respectively). Each spectrum was measured for the direction of the light polarisation maximising the amplitude of the longitudinal resonance. The black line is the ensemble spectrum of the colloidal solution on an arbitrary scale

Similarly the extinction spectrum of a single gold nanorod exhibits two resonances with very different amplitudes [36]. As expected, the red shifted LSPR, with wavelength $\lambda_{\text {SPR }}^{\prime}$ in the $620-800 \mathrm{~nm}$ range, exhibits a very strong light polarisation dependence permitting to define the orientation of the gold nanorod long axis in the substrate plane (Fig. 9). Conversely, only a weak polarisation contrast is observed around the

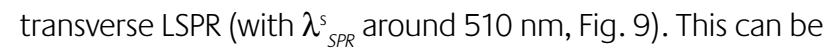
attributed to a main contribution from interband transitions (polarisation independent absorption) that strongly overlap the transverse LSPR in gold nanorods. The measured maximum amplitude of the extinction cross-section is much larger at the longitudinal LSPR than for the transverse LSPR and interband transition region (about 15 times larger for the $\eta \approx 0.5$ nanorod of Fig. 9) making the latter barely visible in the full spectra (Fig. 8). This large contrast is a consequence of the very large enhancement of the longitudinal LSPR crosssection, in agreement with theoretical models (see below). Note that in scattering based methods, only the longitudinal LSPR of a single nanorod has been observed [40,41]. This is due to the fact that even for relatively large rods as investigated here, the transverse resonance is associated to a very weak light scattering, its absorption strongly dominating its optical response $\left(\sigma_{\mathrm{sca}} / \sigma_{\mathrm{abs}} \approx 0.05\right.$ and 0.25 at the transverse and longitudinal LSPR wavelength, respectively) [36].

As SMS measurements are quantitative and yield access to all the spectral feature of the nanorod optical response (its transverse and longitudinal LSPR as well as its interband absorption), the results can be precisely compared to theoretical calculations [31,39]. The optical properties being

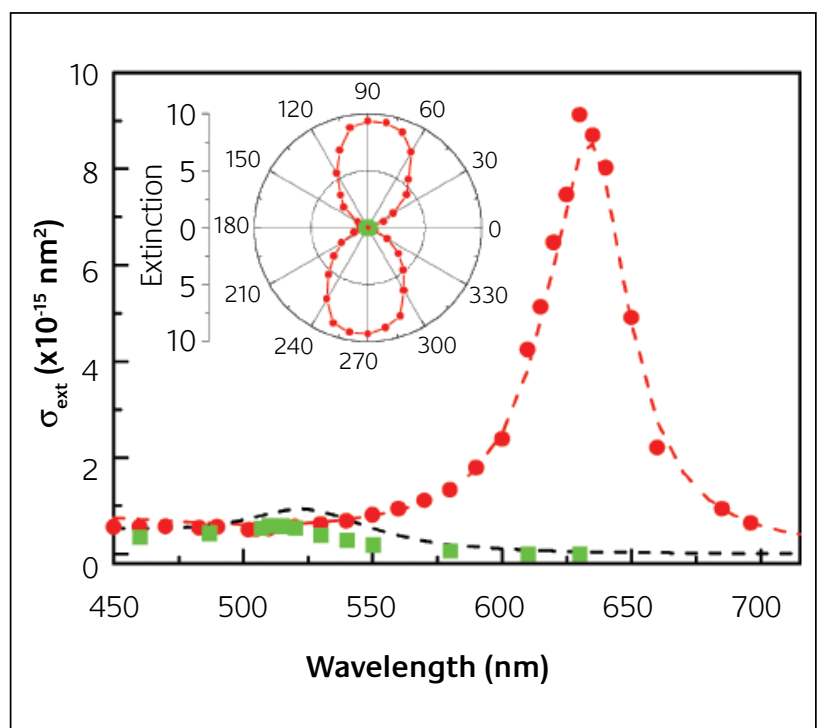

\section{Figure $\mathbf{9}$}

Measured extinction cross section $\sigma_{\text {ext }}$ of a single nanorod for two linear polarisations parallel (red dots) or perpendicular (green squares) to its main axis. The dashed lines are the corresponding computed $\sigma_{\text {ext }}$ assuming a cigar-like shape with an aspect ratio $\eta \approx 0.5$ and length $L$ $=50 \mathrm{~nm}$. The insert shows a polar plot of the measured polarisation dependence of $\sigma_{\text {ext }}$ close to the longitudinal $\lambda=640 \mathrm{~nm}$ (red) and transverse $\lambda=525 \mathrm{~nm}$ (green) LSPR

strongly particle shape sensitive, the model nanorod shape has to correctly mimic the mean one observed with TEM. For the present nanorod a cigar-like shaped particle formed by a cylinder of length / and diameter $D$ capped by hemispheres of radius $D / 2$ has been used [31]. As the optical response cannot be calculated analytically for such shape, modelling has to be performed numerically using Discrete Dipole Approximation (DDA) or Finite Element Method, both approaches permitting description of an arbitrary shape nanoobject $[8,42,43]$. Similar results were obtained using either method, with excellent reproduction of the spectral position, width and amplitude of the longitudinal resonance and slight deviation for the transverse one (using only the particle volume and aspect ratio as parameters). This mainly lies in overestimation of the computed extinction crosssection of the transverse LSPR as compared to the longitudinal one (about 9 times smaller for the transverse than for the longitudinal SPR, the interband absorption, around $475 \mathrm{~nm}$, being about 14 times smaller in agreement with experiments, Fig. 9). This discrepancy was ascribed to deviation of the actual nanorod geometry from the chosen model shape. Actually, the shape of the end caps has an important impact on the LSPR properties and can correct for the above discrepancy [31]. However, more detailed experimentaltheoretical comparison requires more precise definition of the actual nanorod shape, for instance by performing electron microscopy and optical measurements on the same nanoparticle [22,32].

The large sensitivity of the optical response of a nanorod on its geometry (volume, aspect ratio and tip shape) reflects in rod-to-rod uncorrelated variations of its longitudinal LSPR 
wavelength and amplitude (Fig. 8). In particular, for an ellipsoid of fixed volume, $\sigma\left(\lambda_{\text {SPR }}^{\prime}\right)$ is expected to increase with $\lambda_{\text {SPR }}^{\prime}$, in contrast to the experimental observation (Fig. 8), probably because of the different volume of the particle. This is an important parameter for applications, absorption or field enhancement effects and its spatial extension strongly depending not only on the nanoparticle aspect ratio but also on its full size.

The $\lambda_{\text {SPR }}^{\prime}$ fluctuation leads to a much larger width of the longitudinal LSPR in ensemble measurement, as compared to that in a single nanorod (FWHM of $100 \mathrm{~nm}$ as compared to about $35 \mathrm{~nm}$ at $\lambda_{\text {SPR }}^{\prime} \approx 700 \mathrm{~nm}$, Fig. 8). Defining the quality factor of the longitudinal resonance as the ratio between the LSPR peak wavelength and its width, value ranging from 12 to 23 (increasing with wavelength) were obtained for single nanorods, consistent with earlier dark-field scattering measurements on prolate nanoparticles $[40,41]$. This is much larger than the ensemble one, about 7, confirming the large inhomogeneous LSPR broadening, mostly due to fluctuations in the nanoparticle aspect ratio and environment. This large rod to rod spectral variation is also making difficult quantitative estimation of the linear or nonlinear optical response of a single nanorod from ensemble measurements, even if the density of nanorods is precisely known, stressing the importance of single nanoobject optical studies.

\section{$5 \quad$ Single gold nanoparticle optical nonlinear response}

Optical in-situ detection and characterisation of a single metal nanoparticle through its linear extinction signature opens-up many possibilities for nonlinear optics with a single nanoobject. In particular, its nonlinear optical response or its properties investigated by nonlinear optical spectroscopy could then be quantitatively interpreted in connection with its size and geometry (as illustrated above for its linear optical response). In this context, extension of time-resolved femtosecond nonlinear spectroscopy to single gold nanoparticle is particularly interesting [30]. Similarly to ensemble measurements, it should permit investigation of the physical origin of its nonlinear optical response, and its electronic and vibrational kinetics [44-47]. Note that as for nanoparticle linear optics, ensemble studies yield information average over nanoparticle size, shape and environment dispersions (in the case of nanospheres, this has been circumvented by using samples with small size and shape dispersions and performing experiments in environment independent conditions $[45,46])$. Precise investigation of the impact of the particle shape and environment on its nonlinear optical response and on its electron or vibrational response requires the development of femtosecond spectroscopy of single characterised nanoparticles.

The possibility of time-resolved investigation of single metal nanoparticles has been demonstrated using near field optical microscopy on large gold nanorods [48] and far field

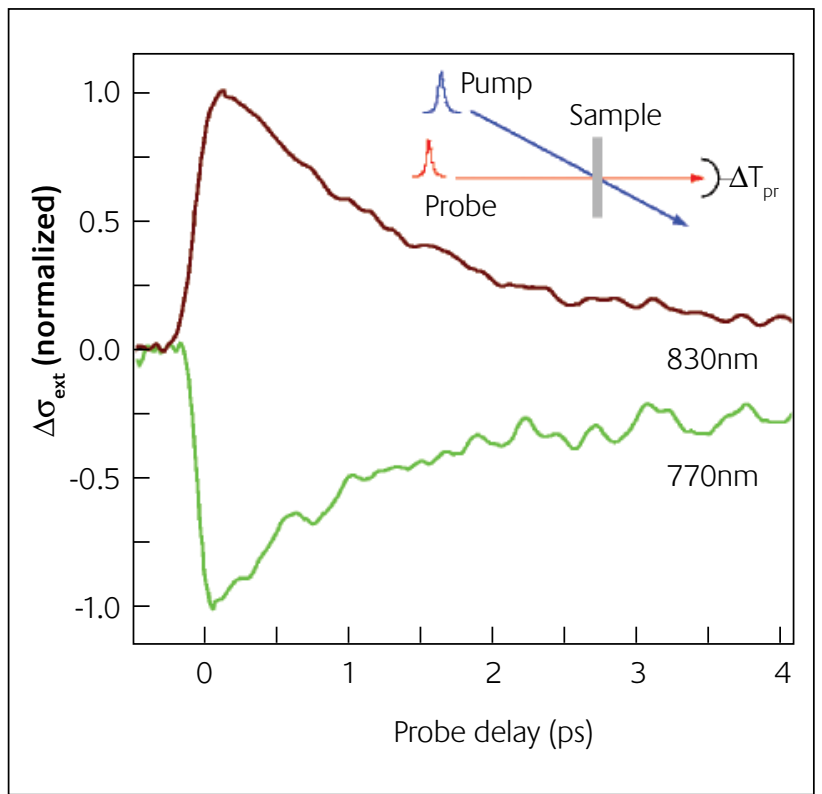

\section{Figure 10}

Transient change of the extinction cross-section $\Delta \sigma_{\text {ext }}\left(\lambda_{\text {probe }}\right)$ of a single gold nanorod measured in a femtosecond pump-probe experiment. The probe wavelength $\lambda_{\text {probe }}$ is either on the blue $(780 \mathrm{~nm})$ or red $(830 \mathrm{~nm})$ side of the longitudinal LSPR of the nanorod $\left(\lambda_{S P R}=795 \mathrm{~nm}\right)$

scattering or absorption based techniques (in sphere with $D \geq 50 \mathrm{~nm}$ ) with picosecond resolution [49,50]. The scattering approach has been recently extended to the femtosecond domain in $80 \mathrm{~nm}$ gold nanospheres and in nanorods [51,52]. However no information on the studied nanoparticle, other than the mean particle size and shape was available. In this context, we have recently shown that, combining SMS technique with a high sensitivity femtosecond pump-probe set-up, nonlinear study of a single silver nanosphere with femtosecond resolution can be performed for size down to $20 \mathrm{~nm}$ [30]. This was optically determined from the single particle absorption spectrum, time-resolved transient absorption measurements being then performed with the same set-up, yielding precise information on the particle electron-lattice coupling. In this type of experiments the transient change of the probe transmission $\Delta T_{p r}$ induced by optical excitation of a single nanoparticle by the pump pulse is monitored (Fig. 10). This is directly proportional to modification of the nanoparticle extinction cross-section $\Delta \sigma_{\text {ext }}\left(\lambda_{\text {probe }}\right)$ permitting its measurement [30]. As in metal films, the measured transient response can thus be connected to the optically induced change of the metal dielectric function, i.e., to a third order nonlinearity of the material $[13,44]$.

We have recently extended this approach to femtosecond investigation of single gold nanorods and single gold prism pairs. The pump-probe delay dependent extinction change $\left.\Delta \sigma_{\text {ext }}\left(\lambda_{\text {probe }}\right)\right)$ of a single gold nanorod with aspect ratio $\eta=0.25$ is illustrated in Fig. 10 . As in ensemble measurements [44], the measured amplitude varies linearly with the pump intensity. Though these results are still preliminary, the opposite sign signals measured for probe 
wavelength $\lambda_{\text {probe }}$ on the red and blue side of the longitudinal LSPR clearly demonstrate that the nonlinear response is dominated by a transient red shift of the LSPR. As in silver nanospheres [44,53], this is tentatively ascribed to increase of the real part 1 of the metal dielectric function due to electron heating by the pump-pulse. It thus corresponds to interaction of the pump and probe pulses via the optical Kerr nonlinearity of the material. As the extinction cross-section of the nanorod at the pump wavelength is known, these measurements permit direct connection between the nonlinear response amplitude and electron temperature rise, and thus quantitative comparison of the experimental data with theoretical modelling of its optical nonlinearity.

\section{Conclusion}

Optical detection and spectroscopy of a single metal nanoparticle with the spatial modulation technique is openingup many possibilities for the investigation of nano-objects. It is presently the only technique permitting quantitative measurement of the extinction cross-section of a single metal nanoparticle. For a small particle, the scattering contribution to extinction can be neglected and the measured signal directly connected to its absorption. This permits full optical determination of its properties: size, shape and orientation, i.e., optical determination of the geometry of an object much smaller than the optical wavelength. The statistics of the results are in very good agreement with the TEM data confirming the validity of this approach. Its present sensitivity of a few $\mathrm{nm}^{2}$ yields a particle size limit of a few nanometer (5 nm for gold). For complex optical signature, combination of this optical investigation with electron microscopy on the same nanoobject, as recently demonstrated for gold nanosphere and dimers [32], is very promising. More generally, this approach can be applied to any absorbing single object.

In situ optical characterisation of a single nanoparticle opens-up many perspectives for the investigation of its properties and their correlation with its geometry and environment. For instance, using the extinction spectra of single nanoparticles as a probe of their local environment, spatial fluctuation of its refractive index has been demonstrated. This opens-up a new way for using characterised single nanoparticles as optical nanoprobes of materials or, for specifically functionalised particles, as nanosensors or biosensors, avoiding the averaging effect inherent to ensemble measurements. This approach is also very interesting as a characterisation tool in systems where electron microscopy cannot be used, as, for instance, for embedded nanoparticles. It also constitutes an important step forward for the analysis of single nanoparticle properties, using nonlinear optical spectroscopy. These non-contact and non-destructive methods are important investigation and characterisation tools of nanomaterials, in particular, in the technologically important situation of nanoobjects in a dielectric matrix.

\section{Acknowledgements}

The authors wish to thank J-R. Huntzinger, P. Billaud, M. Broyer, E. Cottancin, M. Pellarin, J. Lermé, A. Arbouet, O. Muskens, P. Langot, G. Bachelier, A. Brioude, and M.P. Pileni for their essential contributions to part of these studies and for their help in the theoretical and experimental parts of this work. NDF acknowledge support by Institut Universitaire de France.

* Permanent address: Division of Physics, School of Technology, Aristotle University of Thessaloniki, Thessaloniki, 54006, Greece.

\section{About the authors}

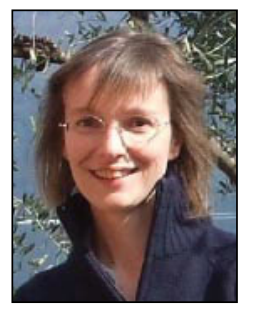

Natalia Del Fatti obtained her Electronic Engineering master degree from Politecnico di Milano (1996), and her Ph.D. in Physics from Ecole Polytechnique, Paris (1999). She was appointed assistant professor at the University of Bordeaux where she worked on ultrafast spectroscopy of metal nanoparticles and developed a novel research axis on single nano-object spectroscopy. Since 2006 she is professor at the University of Lyon, where she co-founded the FemtoNanoOptics group, specialized in optical investigation of nanomaterials. Her current research interests include non-linear optics, ultrafast lasers and nanophysics. She is member of Institut Universitaire de France (IUF).

Natalia Del Fatti, FemtoNanoOptics group, LASIM - CNRSUniversite Lyon 1, 43 Bd. du 11 Novembre 1918, 69622 Villeurbanne-France. Email: delfatti@lasim.univ-lyon1.fr

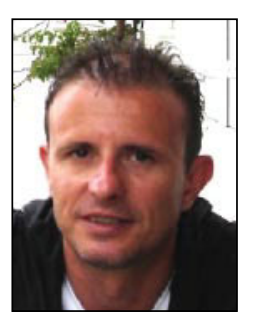

Dimitris Christofilos obtained his degree in Physics in 1990 from the Aristotle University of Thessaloniki (AUTH), Greece and subsequently his masters degree in Electronics\&Radio-electrology and his Ph.D. degree in Physics in 1997 from the same University. He subsequently worked as a post-doctoral fellow in Ecole Polytechnique, Paris (1998-1999), as assistant professor in the Technological Educational Institute of Thessaloniki and as researcher in AUTH (2000-2001) and as an invited CNRS researcher in the University of Bordeaux, France (2002). Since 2003 he has a position in the Faculty of Engineering of AUTH. His research interests include high pressure physics of condensed matter, Raman, absorption and time-resolved spectroscopy. In recent years he has focused his activity on investigation of nanostructures such as carbon nanotubes and metallic nanoparticles. 
Dimitris Christofilos, Physics Division, School of Engineering Aristotle University of Thessaloniki, 54124 Thessaloniki -

Greece. E-mail: christof@vergina.eng.auth.gr

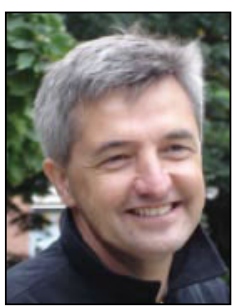

Fabrice Vallée, alumnus of the Ecole Normale Supérieure in Paris, joined the Ecole Polytechnique as a CNRS researcher in 1984 where he worked on nonlinear optics, short pulse lasers and time resolved spectroscopy of solids and nanostructured materials. In 2000 he moved to the University of Bordeaux where he created a new research group in nanophysics and co-founded a multidisciplinary nanosciences and nanotechnology network. He joined the University of Lyon in 2007 where he co-founded the FemtoNanoOptics group specialized in optical investigation of nanomaterials, from a single nano-object to ensembles. His current research interests include nanophysics, nanophotonics and plasmonics, ultrashort pulse lasers, and time resolved optical spectroscopy.

Fabrice Vallée, FemtoNanoOptics group, LASIM - CNRSUniversite Lyon 1, 43 Bd. du 11 Novembre 1918, 69622 Villeurbanne - France. Email: f.vallee@lasim.univ-lyon1.fr

\section{References}

1 M. Faraday, Philos. Trans. R. Soc. (London), 1857, 147, 145

2 G. Mie, Am. Phys. (Leipzig), 1908, 25, 377

3 U. Kreibig and M. Vollmer, Optical Properties of Metal Clusters, Springer Verlag, Berlin 1995 ; U. Kreibig and L. Genzel, Surf. Sci., 1985, 156, 678

4 C.F. Bohren and D.R. Huffman, Absorption and Scattering of Light by Small Particles, John Wiley, New-York,1998

5 M.P. Pileni, CR Chimie, 2003, 6, 965; Nature Materials, 2003, 2, 145

6 L.M. Liz-Marzan, Langmuir, 2006, 22, 32

7 J. Eastoe, M.J. Hollamby and L. Hudson, Adv. Coll. Int. Sci., 2006, 128-130, 5

8 K.L. Kelly, E. Coronado, L.L. Zhao and G.C. Schatz, J. Phys. Chem. B, 2003, 107, 668

9 F. Vallée, in Nanoscience: Nanomaterials and Nanochemestry, C. Dupas, P. Houdy, M. Lahmani, Eds., Springer Verlag, Berlin, 2008, p. 197

10 A.D. McFarland, and R.P. van Duyne, Nano Lett. 2003, 3, 1057

11 G. Raschke, S. Kowarik, T. Franzl, C. Sönnichsen, T.A. Klar, J. Feldmann, A. Nichtl, and K. Kürzinger, Nano Letters, 2003, 3, 935

12 J.J. Mock, D.R. Smith and S. Schultz, Nano Lett., 2003, 3, 485

13 F. Hache, D. Ricard, C. Flytzanis, J. Opt. Soc. Am. B, 1986, 3, 1647 ; C. Flytzanis, F. Hache, M.C. Klein, D. Ricard, and P. Roussignol, In Progress in Optics Vol. XXIX, Ed. E. Wold, North Holland, Amsterdam, 1991, p.321

14 W.E. Moerner and M. Orrit, Science, 1999, 283, 1670

15 S.A. Empedocles, D.J. Norris and M.G. Bawendi, Phys. Rev. Lett., 1996, 77, 3873

16 A. Hartschuh, H.N. Pedrosa, L. Novotny and T.D. Krauss, Science, 2003, 301, 1354

17 T.Klar, M.Perner, S.Grosse, G.Von Plessen, W.Spirkl and J. Feldmann, Phys. Rev. Lett., 1998, 80, 4249
18 A.A. Mikhailovsky, M.A. Petruska, M.I. Stockman and V.I. Klimov, Opt. Lett., 2003, 28, 1686

19 A. Liu, A. Rahmani, G.W. Bryant, L.J. Richter, and S.J. Stranick, J. Opt. Soc. Am. A, 2001, 18, 704

20 A.M. Michaels, M. Nirmal and L.E. Brus, J. Am. Chem. Soc., 1999, 121, 9932

21 C. Sönnichsen, T. Franzl, T. Wilk, G. von Plessen and J. Feldmann, New J. of Phys. 2002, 4, 93

22 J.J. Mock, M. Barbic, D.R. Smith, D.A. Schultz and S. Schultz, J. Chem. Phys., 2002, 116, 6755

23 H. Tamaru, H. Kuwata, H.T. Miyazaki and K. Miyano, Appl. Phys. Lett. 2002, 80, 1826

24 K. Lindfors, T. Kalkbrenner, P. Stoller and V. Sandoghdar, Phys. Rev. Lett., 2004, 93, 37401

25 T. Kalkbrenner, U. Håkanson and V. Sandoghdar, Nano Lett, 2004, 4, 2309

26 D. Boyer, P. Tamarat, A. Maali, B. Lounis and M. Orrit, Science, 2002, 297, 1160

27 A. Arbouet, D. Christofilos, N. Del Fatti, F. Vallée, J-R. Huntzinger, L. Arnaud, P. Billaud and M. Broyer, Phys. Rev. Lett., 2004, 93, 127401

28 O. Muskens, D. Christofilos, N. Del Fatti and F. Vallée, J. Opt. A, 2006, 8, S264

29 O.L. Muskens, N. Del Fatti, F. Vallée, J-R. Huntzinger, P. Billaud and M. Broyer, Appl. Phys. Lett., 2006, 88, 063109

30 O.L. Muskens, N. Del Fatti and F. Vallée, Nano Lett., 2006, 6, 552

31 O. Muskens, G. Bachelier, N. Del Fatti, F. Vallée, A. Brioude, X. Jiang and M.P. Pileni, J. Phys. Chem. C, 2008, to be published

32 P. Billaud, S. Marhaba, E. Cottancin, L. Arnaud, G. Bachelier, C. Bonnet, N. Del Fatti, J. Lermé, F. Vallée, J. -LVialle, M. Broyer and M. Pellarin, J. Phys. Chem. C, 2008, 112, 978

33 P. Billaud, J.-R. Huntzinger, E. Cottancin, J. Lermé, M. Pellarin, L. Arnaud, M. Broyer, N. Del Fatti and F. Vallée, Eur. Phys. J. D, 2007, 43, 271

34 J.K. Ranka, R.S. Windeler and A.J. Stentz, Opt. Lett., 2000, 25, 25

35 P. Langot, N. Del Fatti, R. Tommasi and F. Vallée, Opt. Comm., 1997, 137, 285

36 O.L. Muskens, P. Billaud, M. Broyer, N. Del Fatti and F. Vallée, to be published

37 M.A. El-Sayed and A.S. Majied, Colloids and surfaces A, 2004, 246, 61

38 J. Pérez-Juste, L.M. Liz-Marzan, S. Carnie, D.Y.C. Chan and P. Mulvanay, Adv. Funct. Mater., 2004, 14, 571

39 A. Brioude, X.C. Jiang and M.P. Pileni, J. Phys. Chem B, 2005, 109, 13138

40 C. Sönnichsen, T. Franzl, T. Wilk, G.von Plessen, J. Feldmann O. Wilson and P. Mulvaney, Phys. Rev. Lett., 2002, 88, 077402

41 C. Novo, D. Gomez, D.; J. Perez-Juste, Z. Zhang, H. Petrova, M. Reismann, P. Mulvaney, and G.V. Hartland, Phys. Chem. Chem. Phys., 2006, 8, 3540

42 C. Salzemann, I. Lisiecki, A. Brioude, J. Urban and M.P. Pileni, J. Phys. Chem. B, 2004, 108, 13242

$43 \mathrm{~J}$. Jin, The finite elements method in electrodynamics, Wiley Intersci. New-York, 2002

44 C. Voisin, N. Del Fatti, D. Christofilos and F. Vallée, J. Phys. Chem B, 2001, 105, 2264; N. Del Fatti and F. Vallée, C. R. Acad. Sci., 2002, 3, 365

45 C. Voisin, D. Christofilos, P.A. Loukakos, N. Del Fatti, F. Vallée, J. Lermé, M. Gaudry, E. Cottancin, M. Pellarin and M. Broyer, Phys. Rev. B, 2004, 69, 195416

46 A. Arbouet, C. Voisin, D. Christofilos, P. Langot, N. Del Fatti, F. Vallée, J. Lermé, G. Celep, E. Cottancin, M. Gaudry, M. Pellarin, M. Broyer, M. Maillard, M.P. Pileni, and M.Treguer, Phys. Rev. Lett., 2003, 90, 177401 
C. Voisin, N. Del Fatti, D. Christofilos and F. Vallée, Appl. Surface Science, $2000,164,131$

48 K. Imura, T. Nagahara and H. Okamoto, J. Phys. Chem. B, 2004, 108, 16344

49 Y. Matsuo and K. Sasaki, Japan. J. Appl. Phys. 2001, 40, 6143

50 M.A.van Dijk, M. Lippitz, and M. Orrit, Phys. Rev. Lett., 2005, 95, 267406

51 T. Itoh, T. Asahi and H. Masuhara, Appl. Phys. Lett., 2001, 79, 1667

52 M.Pelton, M.Liu, S.Park, N.F.Scherer and P.Guyot-Sionnest, Phys. Rev. B, 2006, 73, 155419

53 N. Del Fatti, F. Vallée, C. Flytzanis, Y. Hamanaka and A. Nakamura, Chem. Phys., 2000, 251, 215 